\title{
Role of the Low-Density Lipoprotein-Cholesterol/ High-Density Lipoprotein-Cholesterol Ratio in Predicting Serial Changes in the Lipid Component of Coronary Plaque
}

\author{
Ryo Kawakami, MD; Ichiro Matsumoto, MD; Motoi Shiomi, BSc; \\ Mizuki Kurozumi, MD, PhD; Yuichi Miyake, MD, PhD; Makoto Ishizawa, MD, PhD; \\ Kaori Ishikawa, MD, PhD; Kazushi Murakami, MD, PhD; Takahisa Noma, MD, PhD; \\ Yuichiro Takagi, MD, PhD; Naoki Nishimoto, PhD; Tetsuo Minamino, MD, PhD
}

\begin{abstract}
Background: The lipid component of coronary plaques is associated with their vulnerability. The aim of this study was to investigate which coronary risk factors were relevant in predicting serial changes in the lipid component of coronary plaques as evaluated by integrated backscatter intravascular ultrasound (IB-IVUS).
\end{abstract}

\begin{abstract}
Methods and Results: We enrolled 104 patients who underwent IB-IVUS-guided percutaneous coronary intervention (PCI) and were followed up with repeat IB-IVUS 6 months later. We investigated the serial changes in the plasma lipoprotein levels and the percentage of the lipid component of coronary plaques on IB-IVUS. In the multivariate linear regression analysis, the low-density lipoprotein-cholesterol/high-density lipoprotein-cholesterol $(\mathrm{L} / \mathrm{H})$ ratio independently had a significant fixed effect with the percentage of the lipid component of coronary plaques at the time of $\mathrm{PCl}$. In addition, the change in the $\mathrm{L} / \mathrm{H}$ ratio at the 6-month follow-up was significantly associated with that in the lipid component of coronary plaques (regression coefficient, 9.645; 95\% Cl: 5.814-13.475; $\mathrm{P}<0.0001)$; furthermore, this change was also observed in patients with an LDL-C $<100 \mathrm{mg} / \mathrm{dL}$.
\end{abstract}

Conclusions: The $\mathrm{L} / \mathrm{H}$ ratio was the most relevant parameter in predicting the lipid component of coronary plaques. Furthermore, strict management of the $\mathrm{L} / \mathrm{H}$ ratio may reduce this lipid component, even in patients with an LDL-C $<100 \mathrm{mg} / \mathrm{dL}$.

Key Words: Hypercholesterolemia; Intravascular ultrasound; Lipoproteins; Vulnerable plaque

I $\mathrm{n}$ the past decade, cardiovascular disease (CVD) has emerged as the leading cause of death worldwide. Most cases of acute coronary syndrome (ACS), which is one of the most traumatic events in CVD, are triggered by the rupture of a vulnerable plaque followed by thrombosis formation at the rupture site.,1,2 Vulnerable plaques are characterized by a large, lipid-enriched necrotic core overlaid with a thin fibrous cap. ${ }^{3}$ Pathological studies have revealed that the size of the lipid component of coronary plaques is strongly associated with their vulnerability. ${ }^{\mathbf{6}}$ Integrated backscatter intravascular ultrasound (IB-IVUS) is capable of assessing the lipid component of coronary plaques and can evaluate serial changes in the lipid component during drug interventions., ${ }^{5,7-9}$

There are many risk factors for the development of CVD; hypertension, dyslipidemia, diabetes, obesity, and cigarette smoking are traditional systemic risk factors, ${ }^{\mathbf{1 0}}$ and the low-density lipoprotein-cholesterol (LDL-C) level is a well-established molecular risk factor. ${ }^{11}$ A meta-analysis of 4 randomized trials using conventional IVUS revealed that a decrease in both the LDL-C level and the LDL-C/ high-density lipoprotein-cholesterol (HDL-C) ratio (L/H ratio) after statin treatment was associated with regression of the total atheroma volume in patients with CVD. ${ }^{\mathbf{1 2}}$ Although the lipid component of coronary plaques as evaluated by IB-IVUS more precisely reflects the vulnerability of coronary plaques and predicts clinical outcomes compared with evaluation of the atheroma volume using conventional IVUS, 7,13 it has not been determined which coronary risk factors are associated with the serial changes in the lipid component of coronary plaques evaluated by IB-IVUS. Accordingly, we sought to investigate this in the

Received November 28, 2016; revised manuscript received March 21, 2017; accepted April 3, 2017; released online April 29, 2017 Time for primary review: 29 days

Department of Cardiorenal and Cerebrovascular Medicine, Faculty of Medicine, Kagawa University, Kagawa (R.K., Y.M., M.I., K.I., K.M., T.N., T.M.); Cardiovascular Center, KKR Takamatsu Hospital, Kagawa (I.M., M.S., M.K., Y.T.); and Clinical Research Support Center, Kagawa University Hospital, Kagawa (N.N.), Japan

Mailing address: Tetsuo Minamino, MD, PhD, Department of Cardiorenal and Cerebrovascular Medicine, Faculty of Medicine, Kagawa University, 1750-1 Ikenobe, Miki-cho, Kita-gun, Kagawa 761-0793, Japan. E-mail: minamino@med.kagawa-u.ac.jp

ISSN-1346-9843 All rights are reserved to the Japanese Circulation Society. For permissions, please e-mail: cj@j-circ.or.jp 


\begin{tabular}{|c|c|c|}
\hline Parameter & $\begin{array}{l}\text { Total patients } \\
\quad(n=104)\end{array}$ & $\begin{array}{l}\text { Patients with LDL-C }<100 \mathrm{mg} / \mathrm{dL} \\
(\mathrm{n}=47)\end{array}$ \\
\hline Mean age (years) & $68.4 \pm 10.0$ & $69.1 \pm 9.4$ \\
\hline Male & $87(83.7)$ & $41(87.2)$ \\
\hline BMI $\left(\mathbf{k g} / \mathbf{m}^{2}\right)$ & $23.9 \pm 3.6$ & $23.8 \pm 3.7$ \\
\hline \multicolumn{3}{|l|}{ Clinical history } \\
\hline Hypertension & $56(53.8)$ & $27(57.4)$ \\
\hline Diabetes & $37(35.6)$ & $19(40.4)$ \\
\hline Smoking & $35(33.7)$ & $19(40.4)$ \\
\hline \multicolumn{3}{|l|}{ Target plaque location } \\
\hline LAD & $52(50.0)$ & $23(48.9)$ \\
\hline LCX & $19(18.3)$ & $8(17.0)$ \\
\hline RCA & $33(31.7)$ & $16(34.0)$ \\
\hline \multicolumn{3}{|l|}{ Medications } \\
\hline Statin & $62(59.6)$ & $35(74.5)$ \\
\hline Antiplatelet agent & $104(100)$ & $47(100)$ \\
\hline ARB/ACEI & $49(47.1)$ & $19(40.4)$ \\
\hline$\beta$-blocker & $24(23.1)$ & $13(27.7)$ \\
\hline $\mathrm{CCB}$ & $27(26.0)$ & $14(30.0)$ \\
\hline
\end{tabular}

Data are expressed as mean \pm standard deviation or number (\%). ARB/ACEI, angiotensin II receptor blocker/angiotensin converting-enzyme inhibitor; BMI, body mass index; CCB, calcium-channel blocker; LAD, left anterior descending; LCX, left circumflex; LDL-C, low-density lipoprotein cholesterol; RCA, right coronary artery.

current study.

\section{Methods}

\section{Study Population}

We prospectively enrolled 141 consecutive patients who underwent successful percutaneous coronary intervention (PCI) after either ACS or stable angina pectoris (SAP) between January 2010 and July 2012 and in whom IBIVUS at the same lesion could be performed twice. We investigated the serum lipid profiles and the percentage of the lipid component of coronary plaques by IB-IVUS at the time of the PCI and 6 months later. The exclusion criteria included residual stenosis (defined as $>50 \%$ of the diameter) in the left main trunk (LMT) as determined by quantitative coronary angiography or a history of either coronary artery bypass grafting or malignant tumor. Additionally, patients who experienced coronary artery events within 3 months after PCI were excluded to avoid the influence of the PCI procedure or subacute thrombosis. Furthermore, patients were also excluded if they had difficulty undergoing IVUS or had $>50 \%$ calcification of the coronary artery wall. After the first PCI, the follow-up and standard drug treatments were initiated in the outpatient clinic. The patients were carefully monitored during their recovery, and their blood samples were collected early in the morning after an overnight fast at least twice: prior to PCI and at the 6-month follow-up. The use of antidyslipidemic drugs was left to the judgment of the attending physician who set no particular restrictions, but we made efforts to achieve and/or maintain optimal lipid levels during the 6-month recovery after PCI. The Ethics Committee of KKR Takamatsu Hospital approved the study protocol, and all patients gave written informed consent prior to enrollment.

\section{Definitions}

The demographic and clinical data, including age, sex, body mass index (BMI), coronary risk factors (hypertension, diabetes, and smoking history), blood parameters (LDL-C calculated using direct methods, HDL-C, total cholesterol [TC], triglycerides [TG], hemoglobin A1c [HbAlc], and C-reactive protein [CRP, calculated using the Schwartz formula]), and medication use were retrospectively collected from the institution's database. Hypertension was defined as systolic blood pressure $>140 \mathrm{mmHg}$, diastolic blood pressure $>90 \mathrm{mmHg}$, and/or an active regimen of oral antihypertensive drugs. Diabetes mellitus was confirmed if patients had an HbA1c level $\geq 6.5 \%$, a history of any antihyperglycemic medication or a previous diagnosis of diabetes.

\section{Measurement of Conventional and IB-IVUS Parameters}

Several previous reports have already established the reliability and utility of IB-IVUS., ${ }^{7,914,15}$ To prevent coronary spasm, we administered an optimal dose of intracoronary isosorbide dinitrate before obtaining the measurements. The catheter was attached to an IVUS imaging system (VISIWAVE, Terumo, Japan) with automatic pullback at $0.5 \mathrm{~mm} / \mathrm{s}$. Candidate plaques for imaging were selected from the proximal site of the main branch where the last 5 $\mathrm{mm}$ from the stent edge was excluded. Imaging was performed at the site of the same lesion 6 months after PCI. For the purpose of minimizing measurement errors, data collection from both conventional IVUS and IB-IVUS was performed by a single skilled medical engineer. A total of 10 cross-sectional images precisely spaced $0.5 \mathrm{~mm}$ apart were selected for measurement. The leading edge of the lumen and the external elastic membrane were traced using manual planimetry and the plaque area was defined as the area between these leading edges. Because the cross-sectional images were obtained at $0.5-\mathrm{mm}$ intervals, the total plaque volume (TPV) could be calculated using the Simpson 


\begin{tabular}{|c|c|c|c|c|c|c|}
\hline \multirow{2}{*}{ Parameter } & \multicolumn{3}{|c|}{ All patients $(n=104)$} & \multicolumn{3}{|c|}{ Patients with LDL-C <100 mg/dL ( $n=47)$} \\
\hline & Baseline & Follow-up & $P$ value & Baseline & Follow-up & $P$ value \\
\hline \multicolumn{7}{|l|}{ Laboratory data } \\
\hline Total-C (mg/dL) & $186.0 \pm 40.6$ & $167.8 \pm 31.2$ & $<0.001$ & $156.1 \pm 24.8$ & $160.9 \pm 30.8$ & 0.3095 \\
\hline LDL-C (mg/dL) & $110.7 \pm 33.9$ & $90.8 \pm 23.6$ & $<0.0001$ & $82.2 \pm 14.0$ & $84.3 \pm 22.8$ & 0.4871 \\
\hline $\mathrm{HDL}-\mathrm{C}(\mathrm{mg} / \mathrm{dL})$ & $50.5 \pm 15.4$ & $52.0 \pm 15.4$ & 0.4959 & $47.0 \pm 12.4$ & $50.1 \pm 13.5$ & 0.0515 \\
\hline $\mathrm{L} / \mathrm{H}$ ratio & $2.3 \pm 0.8$ & $1.9 \pm 0.6$ & $<0.0001$ & $1.8 \pm 0.5$ & $1.8 \pm 0.6$ & 0.3415 \\
\hline Non-HDL-C (mg/dL) & $135.5 \pm 36.3$ & $115.8 \pm 26.7$ & $<0.0001$ & $109.1 \pm 21.2$ & $110.7 \pm 28.1$ & 0.6800 \\
\hline $\mathrm{TG}(\mathrm{mg} / \mathrm{dL})$ & $135.0 \pm 69.8$ & $149.6 \pm 77.1$ & 0.1553 & $141.8 \pm 73.0$ & $161.5 \pm 87.9$ & 0.1099 \\
\hline $\mathrm{HbA1c}(\%)$ & $6.1 \pm 1.2$ & $6.1 \pm 1.0$ & 0.7244 & $6.1 \pm 1.1$ & $6.1 \pm 0.9$ & 0.8679 \\
\hline $\mathrm{CRP}(\mathrm{mg} / \mathrm{dL})$ & $0.23 \pm 0.49$ & $0.19 \pm 0.51$ & 0.5081 & $0.32 \pm 0.71$ & $0.26 \pm 0.73$ & 0.6981 \\
\hline \multicolumn{7}{|l|}{ Conventional IVUS } \\
\hline Total vessel volume $\left(\mathrm{mm}^{3}\right)$ & $78.0 \pm 23.2$ & $73.8 \pm 23.4$ & 0.1984 & $74.3 \pm 21.5$ & $70.3 \pm 22.3$ & 0.0716 \\
\hline Total lumen volume $\left(\mathrm{mm}^{3}\right)$ & $43.3 \pm 20.3$ & $39.8 \pm 19.3$ & 0.2086 & $38.1 \pm 18.8$ & $35.8 \pm 17.6$ & 0.2372 \\
\hline $\operatorname{TPV}\left(\mathrm{mm}^{3}\right)$ & $34.7 \pm 11.2$ & $34.0 \pm 11.7$ & 0.6590 & $36.3 \pm 10.5$ & $34.5 \pm 12.3$ & 0.1727 \\
\hline Plaque burden (\%) & $46.5 \pm 14.7$ & $47.9 \pm 15.5$ & 0.3204 & $51.1 \pm 15.2$ & $50.2 \pm 14.6$ & 0.6620 \\
\hline \multicolumn{7}{|l|}{ IB-IVUS } \\
\hline $\operatorname{TLV}\left(\mathrm{mm}^{3}\right)$ & $9.5 \pm 5.4$ & $7.5 \pm 5.8$ & $<0.05$ & $9.0 \pm 5.3$ & $7.5 \pm 5.6$ & 0.1547 \\
\hline Percentage of lipid volume (\%) & $26.5 \pm 11.8$ & $20.3 \pm 11.6$ & $<0.001$ & $23.8 \pm 11.2$ & $20.3 \pm 12.1$ & 0.1378 \\
\hline $\operatorname{TFV}\left(\mathrm{mm}^{3}\right)$ & $23.3 \pm 7.8$ & $24.1 \pm 7.6$ & 0.4274 & $25.1 \pm 7.3$ & $24.4 \pm 8.4$ & 0.4852 \\
\hline Percentage of fibrous volume (\%) & $67.6 \pm 9.6$ & $72.3 \pm 9.0$ & $<0.001$ & $70.1 \pm 9.2$ & $71.8 \pm 9.8$ & 0.4009 \\
\hline
\end{tabular}

Data are expressed as mean \pm standard deviation or number (\%). CRP, C-reactive protein; HDL-C, high-density lipoprotein-cholesterol; IB, integrated backscatter; IVUS, intravascular ultrasound; LDL-C, low-density lipoprotein-cholesterol; L/H ratio, LDL-C/HDL-C ratio; TC, total cholesterol; TFV, total fibrous volume; TG, triglycerides; TLV, total lipid volume; TPV, total plaque volume.

method as the mean plaque area multiplied by the pullback length in millimeters. IB signals were obtained using a commercially available system connected to the IVUS imaging system (IB-IVUS, YD Co., Ltd., Nara, Japan) and a 40-MHz IVUS catheter. The IB values for each tissue component were defined as the average power of the backscattered ultrasound signal. The IB values were classified into 4 color maps for each histological category: fibrosis, dense fibrosis, lipid, and calcification area, indicated as green, yellow, blue, and red, respectively. The percentages of each colored area were automatically calculated, and the total lipid volume (TLV) and total fibrous volume (TFV) were determined as the sum of the lipid and fibrous areas, respectively, in each cross-sectional area at $0.5-\mathrm{mm}$ axis intervals. The percentage of the lipid component was calculated as TLV/TPV. The distribution of the lesions might depend on the distance from main branches and the diameter of the coronary artery. To prevent extraneous measurement of coronary compliance, we first selected the proximal site of the main branch and performed a volumetric analysis to assess the plaque characteristics. Second, all measurements were performed 3-5 min after the administration of isosorbide dinitrate because the nitroglycerin-induced effect on coronary distensibility lasts for at least 2 min. ${ }^{\mathbf{1 6}}$ Additionally, IB-IVUS cannot determine the tissue components underlying any calcification because the ultrasound signal cannot penetrate calcium, which is why we excluded patients with severely calcified lesions. Therefore, our results cannot be extrapolated to patients with heavily calcified coronary plaques.

\section{Statistical Analysis}

The data are expressed as the mean \pm standard deviation for continuous variables. Differences in volume between the baseline and 6-month follow-up measurements were assessed with a paired t-test for continuous variables. We performed a univariate linear regression analysis to determine the relationship between the percentage of the lipid component of coronary plaques and each lipid profile. A multivariate linear regression analysis adjusted for age, $\mathrm{BMI}$, hypertension, diabetes, the $\mathrm{L} / \mathrm{H}$ ratio, and $\mathrm{TG}$ was performed to determine the relationship of these parameters to the lipid component of coronary plaques. In order to determine whether LDL-C or HDL-C modifies the associations of the $\mathrm{L} / \mathrm{H}$ ratio with the percentage of the lipid component of coronary plaques, we included a baseline adjustment of LDL-C or HDL-C in the models. In this analysis, the $\mathrm{L} / \mathrm{H}$ ratio was used as a continuous variable. A P-value $<0.05$ was considered to be statistically significant. All data were analyzed using SAS software version 9.1 (SAS Institute Inc., Cary, NC, USA).

\section{Results}

\section{Patients' Characteristics}

Of the 141 patients initially considered, 15 met the exclusion criteria (residual stenosis in LMT, 4; history of coronary artery bypass grafting, 4; malignant tumor, 7). Two patients were excluded because of coronary artery events within 3 months after PCI. We attempted to perform IVUS imaging in the remaining 124 patients prior to PCI, but 15 patients had difficulty with the use of the IVUS catheter, and 5 patients had $>50 \%$ calcification of their coronary artery wall; the remaining 104 patients were eligible for this study.

The baseline clinical characteristics of all the study patients are shown in Table 1. The mean age was $68.4 \pm 10.0$ years, and the majority of the patients were male. Of the 


\begin{tabular}{|c|c|c|c|c|}
\hline & $\begin{array}{l}\text { Regression } \\
\text { coefficient }\end{array}$ & $95 \% \mathrm{Cl}$ & t value & $P$ value \\
\hline $\mathrm{L} / \mathrm{H}$ ratio & 5.681 & $2.987 \sim 8.375$ & 4.18 & $<0.0001$ \\
\hline HDL-C & -0.212 & $-0.357 \sim-0.067$ & -2.91 & $<0.01$ \\
\hline Non-HDL-C & 0.085 & $0.023 \sim 0.146$ & 2.73 & $<0.01$ \\
\hline LDL-C & 0.072 & $0.005 \sim 0.139$ & 2.12 & $<0.05$ \\
\hline TC & 0.037 & $-0.019 \sim 0.094$ & 1.32 & 0.19 \\
\hline TG & 0.035 & $0.002 \sim 0.067$ & 2.11 & $<0.05$ \\
\hline
\end{tabular}

$\mathrm{Cl}$, confidence interval. Other abbreviations as in Table 2.

\begin{tabular}{|c|c|c|c|c|c|}
\hline A & $\begin{array}{l}\text { Regression } \\
\text { coefficient }\end{array}$ & $95 \% \mathrm{Cl}$ & $\begin{array}{l}\text { Standardized partial } \\
\text { regression coefficient }\end{array}$ & $t$ value & $P$ value \\
\hline Age & -0.194 & $-0.422 \sim 0.034$ & -0.164 & -1.69 & 0.095 \\
\hline BMI & -0.329 & $-0.952 \sim 0.294$ & -0.100 & -1.05 & 0.297 \\
\hline Hypertension & -1.276 & $-5.737 \sim 3.185$ & -0.054 & -0.57 & 0.572 \\
\hline Diabetes & 3.058 & $-1.491 \sim 7.606$ & 0.125 & 1.33 & 0.185 \\
\hline L/H ratio & 6.842 & $2.937 \sim 10.748$ & 0.461 & 3.48 & $<0.001$ \\
\hline TG & 0.028 & $-0.004 \sim 0.061$ & 0.167 & 1.72 & 0.088 \\
\hline LDL-C & -0.044 & $-0.135 \sim 0.048$ & -0.126 & -0.95 & 0.344 \\
\hline \multicolumn{6}{|l|}{ B } \\
\hline Age & -0.186 & $-0.411 \sim 0.040$ & -0.157 & -1.63 & 0.106 \\
\hline BMI & -0.341 & $-0.965 \sim 0.283$ & -0.104 & -1.08 & 0.281 \\
\hline Hypertension & -1.373 & $-5.822 \sim 3.076$ & -0.058 & -0.61 & 0.542 \\
\hline Diabetes & 3.071 & $-1.483 \sim 7.624$ & 0.125 & 1.34 & 0.184 \\
\hline $\mathrm{L} / \mathrm{H}$ ratio & 4.861 & 1.632 8.089 & 0.327 & 2.99 & $<0.01$ \\
\hline TG & 0.028 & $-0.005 \sim 0.061$ & 0.166 & 1.69 & 0.093 \\
\hline HDL-C & -0.071 & $-0.235 \sim 0.093$ & -0.092 & -0.86 & 0.395 \\
\hline
\end{tabular}

Covariates: age, BMI, hypertension, diabetes, L/H ratio, TG at follow-up. Abbreviations as in Table 1-3.

\begin{tabular}{|c|c|c|c|c|}
\hline & $\begin{array}{l}\text { Regression } \\
\text { coefficient }\end{array}$ & $95 \% \mathrm{Cl}$ & $t$ value & $P$ value \\
\hline $\mathrm{L} / \mathrm{H}$ ratio & 9.645 & $5.814 \sim 13.475$ & 4.99 & $<0.0001$ \\
\hline HDL-C & -0.461 & $-0.786 \sim-0.136$ & -2.81 & $<0.01$ \\
\hline Non-HDL-C & 0.082 & $0.001 \sim 0.163$ & 2.02 & $<0.05$ \\
\hline LDL-C & 0.088 & $0.002 \sim 0.175$ & 2.02 & $<0.05$ \\
\hline TC & 0.045 & $-0.029 \sim 0.120$ & 1.21 & 0.230 \\
\hline TG & -0.003 & $-0.047 \sim 0.041$ & -0.14 & 0.890 \\
\hline
\end{tabular}

Abbreviations as in Tables 2,3.

104 patients, $62(59.6 \%)$ were treated with statins at baseline, and $89(85.6 \%)$ patients were taking statins at the time of the 6-month follow-up exam. The primary reason why patients were not on a statin regimen at baseline was that they were previous ACS patients and had not visited the hospital for their medical concerns. The laboratory values and measurement of the conventional IVUS and IB-IVUS parameters from the enrolled patients at baseline and at follow-up are summarized in Table 2 . All patients at baseline had higher levels of TC, LDL-C, and non-HDL-C as well as an elevated $\mathrm{L} / \mathrm{H}$ ratio. In the conventional IVUS analysis, there were no significant differences in TPV and plaque burden at follow-up in any of the patients; however, TLV and the percentage of lipid volume were significantly decreased at follow-up in the IB-IVUS analysis.

\section{Factors Associated With the Percentage of the Lipid Component at Baseline}

As shown in Table 3, the univariate linear regression analysis showed that all of the lipid parameters (except TC) 


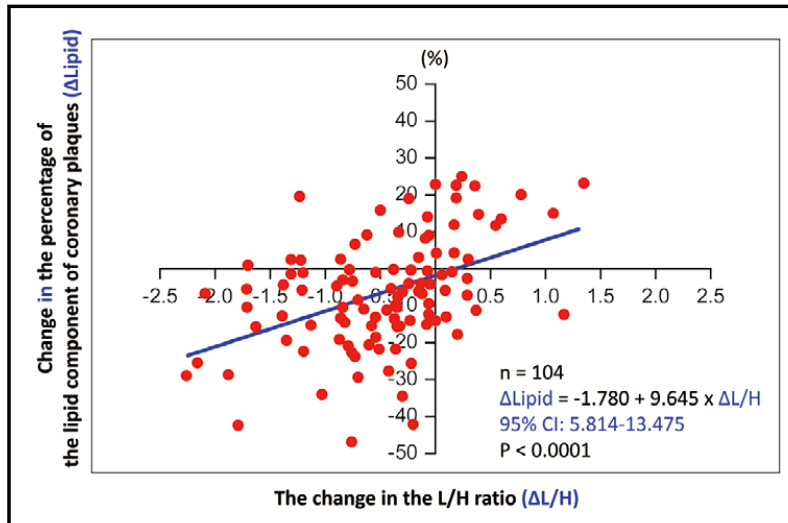

Figure 1. Association between a change in the $\mathrm{L} / \mathrm{H}$ ratio and a change in the percentage of the lipid component of coronary plaques at 6-month follow-up. There was a statistically significant fixed effect between the change in the $\mathrm{L} / \mathrm{H}$ ratio and the change of the percentage of the lipid component of coronary plaques in all the patients enrolled in this study. $\mathrm{L} / \mathrm{H}$, low-density lipoprotein cholesterol/high-density lipoprotein cholesterol ratio.

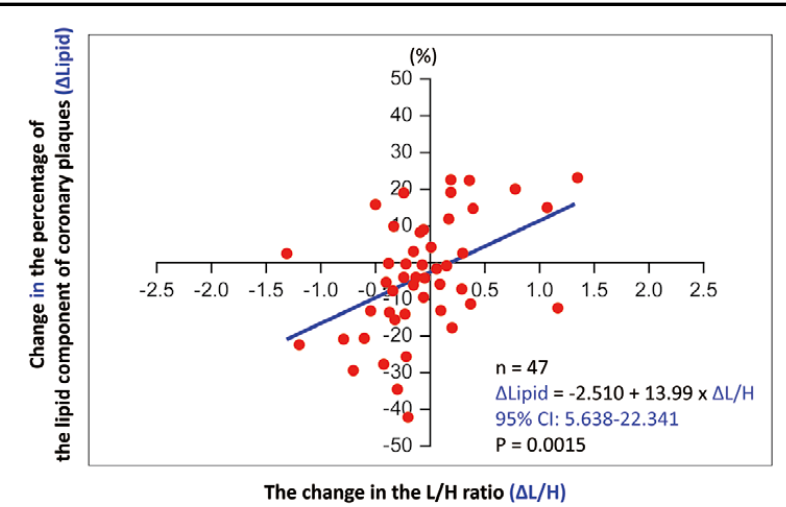

Figure 2. Assessment of 47 patients with LDL-C level $<100 \mathrm{mg} / \mathrm{dL}$ at baseline. Univariate linear regression analysis showed a statistically significant fixed effect between a change in the $\mathrm{L} / \mathrm{H}$ ratio and a change of the percentage of the lipid component of coronary plaques at the 6-month follow-up in these patients. $\mathrm{L} / \mathrm{H}$, low-density lipoprotein cholestero (LDL-C)/high-density lipoprotein cholesterol ratio.

\begin{tabular}{|c|c|c|c|c|}
\hline & $\begin{array}{l}\text { Regression } \\
\text { coefficient }\end{array}$ & $95 \% \mathrm{Cl}$ & t value & $P$ value \\
\hline $\mathrm{L} / \mathrm{H}$ ratio & 13.990 & 5.638 22.341 & 3.37 & $<0.01$ \\
\hline HDL-C & -0.631 & $-1.036 \sim-0.227$ & -3.14 & $<0.01$ \\
\hline TC & -0.093 & $-0.242 \sim 0.056$ & -1.26 & 0.215 \\
\hline TG & -0.021 & $-0.078 \sim 0.037$ & -0.72 & 0.477 \\
\hline Non-HDL-C & -0.029 & $-0.208 \sim 0.150$ & -0.32 & 0.749 \\
\hline LDL-C & -0.017 & $-0.252 \sim 0.217$ & -0.15 & 0.883 \\
\hline
\end{tabular}

Abbreviations as in Tables 2,3.

had a statistically significant fixed effect with the percentage of the lipid component of coronary plaques at baseline. Among the these parameters, the strongest association was observed between the $\mathrm{L} / \mathrm{H}$ ratio and the percentage of the lipid component of coronary plaques (regression coefficient, 5.681; 95\% confidence interval $(95 \% \mathrm{CI}): 2.987-$ 8.375; $\mathrm{P}<0.0001$ ) (Figure S1).

As shown in Table $\mathbf{4 A}$ and Table $\mathbf{4 B}$, we found that the $\mathrm{L} / \mathrm{H}$ ratio independently had a significant increasing effect (regression coefficient, 6.842; 95\% CI: 2.937-10.748; $\mathrm{P}<0.001$ ) for the percentage of the lipid component of coronary plaques using a multivariate linear regression analysis adjusting for TG, confounding factors (age and BMI), coronary risk factors, and LDL-C. Adjusting for HDL-C with the same explanatory variables, the $\mathrm{L} / \mathrm{H}$ ratio had the same increasing effect (regression coefficient, 4.861; 95\% CI: 1.632-8.089; P<0.01). LDL-C and HDL-C, as baseline adjustments in the models, were not statistically significant. Thus, based on the multivariate analysis results we could not confirm whether either of these variables had an effect.

\section{Changes in the Lipid Profile and the Percentage of the Lipid Component at the 6-Month Follow-up}

We repeated the same examinations 6 months after PCI and evaluated the association of the absolute change in each lipid profile with the percentage of the lipid component of coronary plaques. As shown in Table 5, the univariate linear regression analysis showed a statistically significant fixed effect between changes in all of the lipid parameters (except TC and TG) and the percentage of the lipid component of coronary plaques. Among the parameters, the strongest association was observed between changes in the $\mathrm{L} / \mathrm{H}$ ratio and the percentage of the lipid component of coronary plaques (regression coefficient, 9.645; 95\% CI: 5.814-13.475; P<0.0001) (Figure 1).

Furthermore, previous studies have reported that reducing the LDL-C level lowers the risk of CVD-related adverse events. ${ }^{17}$ Therefore, we selected 47 patients with LDL-C $<100 \mathrm{mg} / \mathrm{dL}$ at baseline and analyzed their data to examine the relationship between changes in the $\mathrm{L} / \mathrm{H}$ ratio and the percentage of the lipid component of coronary plaques. Baseline clinical characteristics and the laboratory values and measurement of the conventional and IB-IVUS parameters are summarized in Table $\mathbf{1}$ and Table 2. Although these patients had higher levels of TC, LDL-C, non-HDL$\mathrm{C}$, TLV and the percentage of lipid volume, as well as an elevated $\mathrm{L} / \mathrm{H}$ ratio at baseline, there were no significant differences in these parameters between baseline and follow-up for these patients with LDL-C $<100 \mathrm{mg} / \mathrm{dL}$. Based on these results, the number of patients with increased change in the percentage of the lipid component of coro- 
nary plaques was relatively greater among the patients with LDL-C $<100 \mathrm{mg} / \mathrm{dL}$ rather than among all patients (Figures 1,2). Despite these findings, similar to the results for the entire cohort, there was a statistically significant fixed effect between changes in the $\mathrm{L} / \mathrm{H}$ ratio and the percentage of the lipid component of coronary plaques (regression coefficient, 13.990; 95\% CI: 5.638-22.341; $\mathrm{P}<0.01$ ) (Table 6, Figure 2).

\section{Discussion}

Among the lipid parameters tested, the $\mathrm{L} / \mathrm{H}$ ratio was most strongly associated at both baseline and the 6-month follow-up with the percentage of the lipid component of coronary plaques in patients with coronary artery disease (CAD). After adjusting for various coronary risk factors, multivariate linear regression analysis showed that the $\mathrm{L} / \mathrm{H}$ ratio at baseline remained independently associated with the percentage of the lipid component of coronary plaques. In addition, the changes in the $\mathrm{L} / \mathrm{H}$ ratio and the percentage of the lipid component of coronary plaques during follow-up were positively associated; furthermore, this association was also observed in patients with LDL-C $<100 \mathrm{mg} / \mathrm{dL}$. These findings suggested that the $\mathrm{L} / \mathrm{H}$ ratio had predictive relevance for serial changes in the lipid component of coronary plaques.

Several studies have revealed that disruption of plaque might play a central role in the development of ACS and that plaques with a rich lipid component are more likely to be highly vulnerable to disruption. ${ }^{\mathbf{1 8 - 2 0}}$ Okubo et al previously reported that tissue characterization by IB-IVUS had high sensitivity (90-95\%) and specificity (92-99\%) for histologically identifying the components of plaque. ${ }^{9}$ Furthermore, IB-IVUS imaging of lipid-rich plaque in non-culprit lesions might be a surrogate marker for the development and progression of atherosclerosis and future ischemic events in patients with ACS and SAP. ${ }^{21}$ In this study, we evaluated the lipid component of non-culprit lesions in patients with ACS and SAP. Although some studies have reported that non-culprit lesions in ACS patients had more characteristics of vulnerable plaque and were at higher risk for future adverse events compared with plaques in non-ACS patients, ${ }^{22,23}$ in the present study, the percentage of the lipid component of coronary plaques at baseline, as well as any changes in this percentage at follow-up, did not differ between patients with and without ACS (Table S1). Therefore, we evaluated the relationship between the serum lipid profiles and the lipid component of non-culprit lesions in patients with and without ACS.

Abundant clinical evidence has established that elevated levels of LDL-C constitute a strong predictor of CAD and that LDL-C-lowering therapy is one of the most effective strategies for preventing CVD. ${ }^{17,24-26}$ Plaque regression in the coronary artery as evaluated by conventional IVUS is associated with a decrease in LDL-C levels. ${ }^{12}$ Soeda et al reported that a $45.7 \%$ reduction in LDL-C was accompanied by a $11.3 \%$ decrease in plaque volume, with a concomitant significant decrease in the ratio of the lipid core volume to plaque volume as evaluated by dual-source computed tomography. ${ }^{27}$ Importantly, several epidemiological and clinical studies have reported that the $\mathrm{L} / \mathrm{H}$ ratio rather than individual assessment of LDL-C and HDL-C is more closely associated with the development of CVD. ${ }^{28,29}$ We recently showed that a high $\mathrm{L} / \mathrm{H}$ ratio can affect the clinical outcome of patients after PCI. ${ }^{30}$ Furthermore, an elevated $\mathrm{L} / \mathrm{H}$ ratio is associated with a high lipid component of coronary plaques in patients with SAP in the LMT. ${ }^{31}$ Consistent with this, the present study also confirmed that a high $\mathrm{L} / \mathrm{H}$ ratio is associated with the percentage of the lipid component of coronary plaques (Figure S1, Table 3).

Although previous reports have shown that the $\mathrm{L} / \mathrm{H}$ ratio has a profound effect on the lipid component of coronary plaques, ${ }^{31}$ it remains unknown whether treatment directed at improving the $\mathrm{L} / \mathrm{H}$ ratio is associated with a diminishing lipid component of coronary plaques. In the present study, we performed serial observations of IBIVUS at baseline and at 6-month follow-up and found that the changes in the $\mathrm{L} / \mathrm{H}$ ratio were closely associated with the absolute changes in the percentage of the lipid component of coronary plaques. Furthermore, in order to extend our evaluation of the change in the lipid component of coronary plaques, additional analyses were performed in patients with either decreased or increased changes in the percentage of the lipid component of coronary plaques. Although there were no significant differences (except for smoking) in the baseline clinical characteristics of the 2 groups (Table S2), the percentage of both lipid and fibrous volume showed significant changes during follow-up in both groups as expected (Table S3). Similarly, TC, LDL-C, and non-HDL-C, as well as an elevated $\mathrm{L} / \mathrm{H}$ ratio, at baseline had significantly improved during follow-up in the patients in the decrease group. These findings collectively reflected our main data. Moreover, as there was a significant difference in smoking incidence (Table S2), we performed additional analysis regarding smoking. As shown in Table S4 and Table S5, there were no significant differences (except in the percentage of LAD lesions) between the smokers and non-smokers. Although the baseline levels of HDL-C in the patients who smoked tend to be lower than those in patients who were not smokers, there were no significant changes in HDL-C levels during follow-up in both groups of patients (Table S6). Similar to the results for the entire cohort, our findings showed that changes in the $\mathrm{L} / \mathrm{H}$ ratio showed a significant fixed effect with changes in the lipid component of coronary plaque in nonsmoking patients (regression coefficient, 9.836; 95\% CI: 4.768-14.903; $\mathrm{P}<0.001$ ) (Table S7, Figure S2). Thus, our findings suggested that monitoring changes in the $\mathrm{L} / \mathrm{H}$ ratio would predict changes in the percentage of the lipid component of coronary plaques, which is indicative of plaque vulnerability.

The target of lipid treatment has not been established in patients with CAD. Some guidelines recommended that LDL-C should be lower than $2.5 \mathrm{mmol} / \mathrm{L}(100 \mathrm{mg} / \mathrm{dL})$ for patients with a history of CAD. ${ }^{32-35}$ Recently, a metaanalysis using individual patient data from 8 randomized controlled trials of statin therapy revealed that patients who achieved very low LDL-C levels had a lower risk for major cardiovascular events than did those achieving moderately low levels. ${ }^{36}$ Therefore, additional guidelines also suggest that for patients at high risk for CVD, the level of LDL-C should be strictly lower than $1.8 \mathrm{mmol} / \mathrm{L}(70 \mathrm{mg} / \mathrm{dL})$. In agreement with these data, among the present patients with LDL-C levels $<100 \mathrm{mg} / \mathrm{dL}$, we demonstrated a significant fixed effect between changes in the $\mathrm{L} / \mathrm{H}$ ratio and the percentage of the lipid component of coronary artery plaques (Table 6, Figure 2). Therefore, careful monitoring of the $\mathrm{L} / \mathrm{H}$ ratio, even in patients with an $\mathrm{LDL}-\mathrm{C}<100 \mathrm{mg} / \mathrm{dL}$, is recommended. 


\section{Study Limitations}

There are several potential limitations to note. This study was a post hoc analysis of observational data, which cannot be extrapolated to treatment recommendations. Also, it included a relatively small number of patients at a single center. There are also limitations regarding the implementation of serial IVUS, such as matching the lesions at baseline and 6 months later, non-uniform rotational distortion or a difference in the ultrasound intensity related to the IVUS catheter position. However, we minimized the selection bias by reevaluating the same patients 6 months later and studying only the proximal site of the main branches. Because there may have been circumstances that might introduce bias into the results, additional larger studies are required to validate our findings.

\section{Conclusions}

Our study revealed that the $\mathrm{L} / \mathrm{H}$ ratio was the most relevant factor in predicting the extent of and changes in the lipid component of coronary plaques. Furthermore, patients with a small reduction in the $\mathrm{L} / \mathrm{H}$ ratio should be carefully monitored, even if their LDL-C levels are lower than $100 \mathrm{mg} / \mathrm{dL}$.

\section{Acknowledgments}

We are grateful to Dr. Yasuhiro Ichibori and Dr. Ryo Araki from the Department of Cardiovascular Medicine at the Osaka University Graduate School of Medicine for their valuable suggestions and advice.

\section{Disclosures}

The authors declare no conflicts of interest and no financial support from any institute. All illustrations and figures in the manuscript are entirely original and do not require reprint permission.

\section{Funding Source}

None.

\section{References}

1. Davies MJ, Thomas A. Thrombosis and acute coronary-artery lesion in sudden cardiac ischemic death. $N$ Engl J Med 1984; 310: $1137-1140$.

2. Fuster V, Badimon L, Badimon JJ, Chesebro JH. The pathogenesis of coronary artery disease and the acute coronary syndromes. N Engl J Med 1992; 326: 310-318.

3. Finn AV, Nakano M, Narula J, Kolodgie FD, Virmani R. Concept of vulnerable/unstable plaque. Arterioscler Thromb Vasc Biol 2010; 30: $1282-1292$.

4. Virmani R, Burke AP, Farb A, Kolodgie FD. Pathology of the vulnerable plaque. J Am Coll Cardiol 2006; 47: C13-C18.

5. Kawasaki M, Takatsu H, Noda T, Sano K, Ito Y, Hayakawa K, et al. In vivo quantitative tissue characterization of human coronary arterial plaques by use of integrated backscatter intravascular ultrasound and comparison with angioscopic findings. Circulation 2002; 105: 2487-2492.

6. Bando M, Yamada H, Kusunose K, Fukuda D, Amano R, Tamai R, et al. Comparison of carotid plaque tissue characteristics in patients with acute coronary syndrome or stable angina pectoris: Assessment by iPlaque, transcutaneous carotid ultrasonography with integrated backscatter analysis. Cardiovasc Ultrasound 2015; 13: 34 .

7. Sano K, Kawasaki M, Ishihara Y, Okubo M, Tsuchiya K, Nishigaki $\mathrm{K}$, et al. Assessment of vulnerable plaques causing acute coronary syndrome using integrated backscatter intravascular ultrasound. J Am Coll Cardiol 2006; 47: 734-741.

8. Tanaka S, Noda T, Iwama M, Tanihata S, Kawasaki M, Nishigaki $\mathrm{K}$, et al. Long-term changes in neointimal hyperplasia following implantation of bare metal stents assessed by integrated backscatter intravascular ultrasound. Heart Vessels 2013; 28: 415-423.

9. Okubo M, Kawasaki M, Ishihara Y, Takeyama U, Kubota T,
Yamaki T, et al. Development of integrated backscatter intravascular ultrasound for tissue characterization of coronary plaques. Ultrasound Med Biol 2008; 34: 655-663.

10. Okada K, Hibi K, Gohbara M, Kataoka S, Takano K, Akiyama E, et al. Association between blood glucose variability and coronary plaque instability in patients with acute coronary syndromes. Cardiovasc Diabetol 2015; 14: 111.

11. Kojima S, Kojima S, Maruyoshi H, Nagayoshi Y, Kaikita K, Sumida $\mathrm{H}$, et al. Hypercholesterolemia and hypoadiponectinemia are associated with necrotic core-rich coronary plaque. Int $\mathrm{J}$ Cardiol 2011; 147: $371-376$.

12. Nicholls SJ, Tuzcu EM, Sipahi I, Grasso AW, Schoenhagen P, Hu $\mathrm{T}$, et al. Statins, high-density lipoprotein cholesterol, and regression of coronary atherosclerosis. JAMA 2007; 297: 499-508.

13. Otagiri K, Tsutsui H, Kumazaki S, Miyashita Y, Aizawa K, Koshikawa M, et al. Early intervention with rosuvastatin decreases the lipid components of the plaque in acute coronary syndrome: Analysis using integrated backscatter IVUS (ELAN study). Circ $J$ 2011; 75: 633-641.

14. Kawasaki M, Sano K, Okubo M, Yokoyama H, Ito Y, Murata $\mathrm{I}$, et al. Volumetric quantitative analysis of tissue characteristics of coronary plaques after statin therapy using three-dimensional integrated backscatter intravascular ultrasound. J Am Coll Cardiol 2005; 45: 1946-1953.

15. Iwama M, Tanaka S, Noda T, Segawa T, Kawasaki M, Nishigaki $\mathrm{K}$, et al. Impact of tissue characteristics on luminal narrowing of mild angiographic coronary stenosis: Assessment of integrated backscatter intravascular ultrasound. Heart Vessels 2014; 29: 750760.

16. Yamagishi M, Nissen SE, Booth DC, Gurley JC, Koyama J, Kawano $S$, et al. Coronary reactivity to nitroglycerin: Intravascular ultrasound evidence for the importance of plaque distribution. $J$ Am Coll Cardiol 1995; 25: 224-230.

17. Sakamoto T, Ogawa H. "Just make it lower" is an alternative strategy of lipid-lowering therapy with statins in Japanese patients: LDL-cholesterol: The lower, the better; is it true for Asians? (Con). Circ J 2010; 74: 1731-1741.

18. Stone GW, Maehara A, Lansky AJ, de Bruyne B, Cristea E, Mintz GS, et al. A prospective natural-history study of coronary atherosclerosis. N Engl J Med 2011; 364: 226-235.

19. de Feyter PJ, Ozaki Y, Baptista J, Escaned J, Di Mario C, de Jaegere PP, et al. Ischemia-related lesion characteristics in patients with stable or unstable angina: A study with intracoronary angioscopy and ultrasound. Circulation 1995; 92: 1408-1413.

20. Takaoka N, Tsujita K, Kaikita K, Hokimoto S, Yamanaga K, Komura N, et al. Intravascular ultrasound morphology of culprit lesions and clinical demographics in patients with acute coronary syndrome in relation to low-density lipoprotein cholesterol levels at onset. Heart Vessels 2014; 29: 584-595.

21. Amano T, Matsubara T, Uetani T, Kato M, Kato B, Yoshida T, et al. Lipid-rich plaques predict non-target-lesion ischemic events in patients undergoing percutaneous coronary intervention. Circ J 2011; 75: 157-166.

22. Kato K, Yonetsu T, Kim SJ, Xing L, Lee H, McNulty I, et al. Nonculprit plaques in patients with acute coronary syndromes have more vulnerable features compared with those with nonacute coronary syndromes: A 3-vessel optical coherence tomography study. Circ Cardiovasc Imaging 2012; 5: 433-440.

23. Wald DS, Morris JK, Wald NJ, Chase AJ, Edwards RJ, Hughes LO, et al. Randomized trial of preventive angioplasty in myocardial infarction. N Engl J Med 2013; 369: 1115-1123.

24. Sacks FM, Pfeffer MA, Moye LA, Rouleau JL, Rutherford JD, Cole TG, et al. The effect of pravastatin on coronary events after myocardial infarction in patients with average cholesterol levels: Cholesterol and Recurrent Events Trial investigators. $N$ Engl J Med 1996; 335: 1001-1009.

25. Prevention of cardiovascular events and death with pravastatin in patients with coronary heart disease and a broad range of initial cholesterol levels: The Long-Term Intervention with Pravastatin in Ischemic Disease (LIPID) Study Group. $N$ Engl $J$ Med 1998; 339: 1349-1357.

26. Itakura H, Kita T, Mabuchi H, Matsuzaki M, Matsuzawa Y, Nakaya N, et al. Relationship between coronary events and serum cholesterol during 10 years of low-dose simvastatin therapy: Long-term efficacy and safety in Japanese patients with hypercholesterolemia in the Japan Lipid Intervention Trial (J-LIT) Extension 10 Study, a prospective large-scale observational cohort study. Circ J 2008; 72: 1218-1224.

27. Soeda T, Uemura S, Okayama S, Kawakami R, Sugawara Y, Nakagawa H, et al. Intensive lipid-lowering therapy with rosuv- 
astatin stabilizes lipid-rich coronary plaques: Evaluation using dual-source computed tomography. Circ J 2011; 75: 2621 - 2627.

28. Kannel WB. Risk stratification of dyslipidemia: Insights from the Framingham Study. Curr Med Chem Cardiovasc Hematol Agents 2005; 3: 187-193.

29. Packard CJ, Ford I, Robertson M, Shepherd J, Blauw GJ, Murphy $\mathrm{MB}$, et al. Plasma lipoproteins and apolipoproteins as predictors of cardiovascular risk and treatment benefit in the PROspective Study of Pravastatin in the Elderly at Risk (PROSPER). Circulation 2005; 112: 3058-3065.

30. Matsumoto I, Miyake Y, Mizukawa M, Takagi Y. Impact of low-density lipoprotein cholesterol/high-density lipoprotein cholesterol ratio on long-term outcome in patients undergoing percutaneous coronary intervention. Circ J 2011; 75: 905-910.

31. Kurebayashi N, Yoshikawa D, Ishii H, Sato B, Ando H, Okada $\mathrm{T}$, et al. Impact of the low-to high-density lipoprotein cholesterol ratio on composition of angiographically ambiguous left main coronary artery plaque. Circ J 2011; 75: 1960-1967.

32. Teramoto T, Sasaki J, Ishibashi S, Birou S, Daida H, Dohi S, et al; Japan Atherosclerosis Society. Executive summary of the Japan Atherosclerosis Society (JAS) guidelines for the diagnosis and prevention of atherosclerotic cardiovascular diseases in Japan: 2012 version. J Atheroscler Thromb 2013; 20: 517-523.

33. Smith SC Jr, Allen J, Blair SN, Bonow RO, Brass LM, Fonarow GC, et al. AHA/ACC guidelines for secondary prevention for patients with coronary and other atherosclerotic vascular disease: 2006 update: Endorsed by the National Heart, Lung, and Blood Institute. Circulation 2006; 113: 2363-2372.

34. Brunzell JD, Davidson M, Furberg CD, Goldberg RB, Howard $\mathrm{BV}$, Stein JH, et al. Lipoprotein management in patients with cardiometabolic risk: Consensus statement from the American Diabetes Association and the American College of Cardiology Foundation. Diabetes Care 2008; 31: 811-822.

35. Perk J, De Backer G, Gohlke H, Graham I, Reiner Z, Verschuren $\mathrm{M}$, et al. European Guidelines on cardiovascular disease prevention in clinical practice (version 2012): The Fifth Joint Task Force of the European Society of Cardiology and Other Societies on Cardiovascular Disease Prevention in Clinical Practice (constituted by representatives of nine societies and by invited experts).
Eur Heart J 2012; 33: 1635-1701.

36. Boekholdt SM, Hovingh GK, Mora S, Arsenault BJ, Amarenco $\mathrm{P}$, Pedersen TR, et al. Very low levels of atherogenic lipoproteins and the risk for cardiovascular events: A meta-analysis of statin trials. J Am Coll Cardiol 2014; 64: 485-494.

\section{Supplementary Files}

\section{Supplementary File 1}

Figure S1. The association between the L/H ratio and the percentage of the lipid component of coronary plaques at baseline shows a statistically significant fixed effect.

Figure S2. We assessed 69 patients without smoking.

Table S1. Data for conventional IVUS and IB-IVUS parameters at baseline and 6-month follow-up of patients with and without ACS

Table S2. Baseline clinical characteristics of patients with decreased or increased change in the percentage of the lipid component of coronary plaque

Table S3. Laboratory and imaging data at baseline and follow-up among patients with decreased or increased change in the percentage of the lipid component of coronary plaque

Table S4. Clinical characteristics of the smoking and nonsmoking patients at baseline

Table S5. Laboratory data for the smoking and nonsmoking patients at baseline

Table S6. Laboratory and imaging data at baseline and 6-month follow-up for the smoking and nonsmoking patients

Table S7. Univariate linear regression analysis of the change in the percentage of the lipid component of coronary plaque and each lipid parameter in the nonsmoking patients $(n=69)$ at the 6-month follow-up

Please find supplementary file(s);

http://dx.doi.org/10.1253/circj.CJ-16-1209 